\title{
Desde la concepción de disciplina científica a la noción de didáctica en carreras de la salud
}

\author{
Javiera ORTEGA-BASTIDAS, Olga MATUS-BETANCOURt, Carolina MÁRQUEZ-URRIZOLA, Paula PARRA-PONCE, \\ Débora ALVARADO-FIGUEROA, Cristhian PÉREZ-VILLALOBOS, Juan ARELLANO-VEGA, Nancy BASTÍAS-VEGA
}

Introducción. El estudio de la didáctica en ciencias de la salud ha sido abordado principalmente a través de las innovaciones en metodologías de enseñanza y evaluación. Sin embargo, no se ha realizado una discusión teórico-empírica sobre la naturaleza de la didáctica que constituyen las diversas disciplinas en el área de la salud.

Sujetos y métodos. Estudio cualitativo, basado en la teoría fundamentada. Participaron 31 docentes de seis carreras de ciencias de la salud, a los cuales se seleccionó según el criterio de máxima variación. Los docentes fueron contactados personalmente, previo proceso de consentimiento informado. Se realizaron entrevistas semiestructuradas y grupos focales diseñados por los investigadores. El plan de análisis se realizó a partir del método de comparación constante hasta llegar al nivel de codificación selectiva, utilizando el Caqdas Atlas-ti 7.5.2.

Resultados. El fenómeno se configura en un contexto sociopolítico particular, con condiciones de las carreras de la salud, en un paradigma científico hegemónico. Antecedentes causales: falta de delimitación del objeto de estudio, sus dimensiones y concepciones curriculares. Estrategias de acción: diseño de objetivos, organización de contenidos, metodologías de enseñanza y evaluación, habilidades del docente. Factores intervinientes: rol docente, funcionamiento curricular, características de la disciplina, clima educativo. Consecuencias: positivas y negativas en diseños curriculares, progresión de los aprendizajes y consciencia didáctica.

Conclusión. Los resultados obtenidos evidencian la necesidad de reflexionar acerca de la educación médica, ya que permiten discutir la naturaleza científica y didáctica de las carreras de salud en Chile.

Palabras clave. Ciencia. Didáctica. Investigación cualitativa.

\section{From the conception of scientific discipline to the notion of didactics in health careers}

Introduction. The study of didactics in Health Sciences has been approached mainly through innovations in teaching and evaluation methodologies. However, there has not been a theoretical-empirical discussion on the nature of the didactics that constitute the various disciplines in the area of health.

Subjects and methods. Qualitative study, based on the Grounded Theory. Thirty-one teachers from six health sciences courses participated and were selected according to the criteria of maximum variation. Teachers were contacted in person after the informed consent process. Semi-structured interviews and researchers-designed focus groups were conducted. The analysis plan was carried out from the constant comparison method up to the selective coding level, using the Caqdas Atlas-ti 7.5.2.

Results. The phenomenon is configured in a particular socio-political context, with conditions of health careers, in a hegemonic scientific paradigm. Causal background: lack of delimitation of the object of study, its dimensions and curricular conceptions. Action strategies: design of objectives, organization of contents, teaching and evaluation methodologies, teacher skills. Intervening factors: Teaching role, curricular functioning, characteristics of the discipline, educational climate. Consequences: positive and negative in curricular design, progression of learning, didactic awareness.

Conclusion. The results obtained show the need to reflect on medical education, since they allow us to discuss the scientific and didactic nature of health careers in Chile.

Key words. Didactics. Qualitative research. Science.
Departamento de Educación Médica. Facultad de Medicina. Universidad de Concepción. Concepción, Chile.

Correspondencia:

Profa. Javiera Ortega Bastidas. Departamento de Educación Médica. Facultad de Medicina. Universidad de Concepción. Barrio Universitario, $\mathrm{s} / \mathrm{n}$. CP 4030000. Concepción, Chile.

E-mail:

javieraortega@udec.cl

Financiación:

Proyecto VRID n.ำ 214.090.004-10, Vicerrectoría de Investigación, Universidad de Concepción. Apoyo financiero para la ejecución del proyecto 'Dimensión didáctica de los escenarios educativos en carreras en ciencias de la salud'.

Recibido:

31.10.18.

Aceptado: 06.11.18.

Conflicto de intereses: No declarado.

Competing interests: None declared.

(c) 2019 FEM

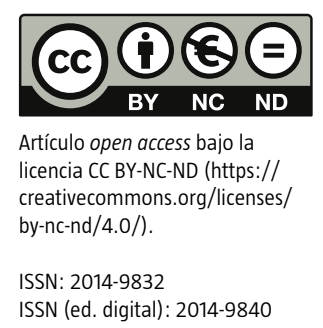




\section{Introducción}

Flexner [1] sentó las bases de la educación médica a principios del siglo $\mathrm{xx}$ y su propuesta ha permitido establecer algunos principios fundamentales en la formación en salud, particularmente la diferenciación entre las ciencias básicas y las clínicas. A pesar de existir propuestas sobre la importancia de pensar un currículo integrado [2], estudios respecto a dicha materia han evidenciado que los docentes de carreras de salud en Chile, al sumarse a los principios flexnerianos, han concebido los diseños curriculares desde una noción que dicotomiza la teoría de la práctica [3]. Asimismo, han sostenido ciertas concepciones técnicas de la enseñanza y la evaluación $[4,5]$. Sin embargo, una discusión a la cual invita este estudio es a reflexionar no tanto sobre cómo los docentes deberían pensar la enseñanza y la evaluación, sino respecto a una cuestión de suma importancia que se ha instituido en las carreras de salud, y es la de su carácter científico. Esto adquiere relevancia en un contexto donde las universidades, como instituciones promotoras de ciencia y formación profesional, se ven cada vez más enfrentadas a constantes cambios tecnológicos, económicos y sociales, lo que obliga a permanecer atentos a dichas transformaciones [6]. En un escenario de esta naturaleza, la importancia en la formación no sólo está enfocada en identificar aquellas formas de acceder al conocimiento, sino que también adquiere una función reflexiva, considerada fundamental en el proceso de adquisición de actitudes, habilidades y competencias que permiten la apropiación teórica y práctica del conocimiento necesario para el ejercicio profesional $[7,8]$. La función que cumple el docente en dicha formación es de vital importancia, puesto que hoy se le atribuye una doble tarea, enseñar e investigar; por tanto, no solo debe saber cómo enseñar [9], sino que debe ser capaz de reconocer el objetivo principal de la disciplina que enseña, sus leyes básicas, aquellas teorías implícitas que la caracterizan y, por consiguiente, su práctica metodológica [10]. Al respecto, Grossman et al [10] proponen que es necesario especificar aquellos principios didácticos que sean particulares para cada disciplina, puesto que cada una de ellas se reviste de una naturaleza ontológica y epistémica particular. En este sentido, la acción del docente cumple un papel fundamental porque es quien debería ejercer la tarea de vincular el conocimiento de su profesión en el proceso educativo. Chevallard [11] fue claro al respecto y enfatizó que es el docente quien debe realizar dicha función a través del proceso de transposición didáctica.
La vinculación que debe hacer el docente trasciende su carácter experiencial, pues en definitiva sería aquel conocimiento propio de su disciplina el que debe articular en su enseñanza [10,11]. En un escenario tal, y considerando que la ciencia es el eje central de la educación superior, este estudio pretende develar aquellos significados que están asociados al pensamiento disciplinar en carreras de la salud y la naturaleza didáctica que ello acarrea. Esto permitirá ir ganando lucidez respecto a cuál es la discusión científica actual en cada disciplina, dónde se ha generado dicha discusión y hacia dónde debería encaminarse. De esta forma, en el doble acto de educar e investigar, la educación superior solo podrá cumplir la misión de formar profesionales competentes que atiendan a las necesidades circundantes en tanto se consagre, al mismo tiempo, a la doble tarea de investigar y enseñar $[7,8,12]$.

\section{Sujetos y métodos}

Este estudio es una investigación cualitativa basada en la teoría fundamentada de Strauss y Corbin [13], perspectiva de análisis inductiva, a través de la cual los datos, el análisis y la teoría logran interrelacionarse con la finalidad de construir, en conjunto con los participantes, sus experiencias y el significado que atribuyen al objeto de estudio [14]. Como técnica de recogida de datos se utilizó la entrevista semiestructurada y el focus group [15]. El análisis de datos se realizó a partir del método de comparación constante [13] hasta el nivel de codificación selectiva, el cual permite construir un modelo integral general que interrelaciona los aspectos principales de los resultados en torno al fenómeno central y a los objetivos propuestos [13]. Los resultados a presentar se construyen a partir de una serie de análisis realizados. Estos estudios serán contrastados constantemente con dichos resultados, puesto que son parte importante de los ejes analíticos para la codificación selectiva.

Los participantes fueron 30 docentes de seis carreras de ciencias de la salud (medicina, enfermería, cinesiología, fonoaudiología, tecnología médica y obstetricia y puericultura de una universidad tradicional chilena) y un experto en didáctica profesional de la pedagogía. Los sujetos se seleccionaron según el criterio de máxima variación de Patton [16] hasta alcanzar la saturación de datos. Los informantes clave fueron los directores de las carreras estudiadas, previo proceso de consentimiento informado institucional y de la carrera. Los participantes tenían una edad media de 43,6 \pm 12,4 años (rango: 26-69 años). 
De ellos, un $38,7 \%(n=12)$ correspondían a hombres y un $61,3 \%(n=19)$ eran mujeres. Del total de entrevistados, un 19,4\% $(n=6)$ impartía docencia clínica; un $48,4 \%(n=15)$, docencia en aula, y un $32,3 \%(n=10)$, en ambos contextos. Los criterios de inclusión fueron que el docente perteneciera a las carreras del área de salud y que impartiera clases en el ciclo básico o ciclo preclínico y clínico. Para delimitar la muestra, se utilizó el muestreo teórico, cuyo criterio básico de selección es la relevancia teórica que tiene el caso para el desarrollo de las categorías emergentes, donde es fundamental considerar participantes que tengan un potencial de información [14]. Se contactó a los participantes a través de los directores de carrera, previo proceso de consentimiento informado. Como es característico del paradigma cualitativo, se salvaguardaron los criterios de rigor científico, como credibilidad, transferibilidad y confirmabilidad [17].

\section{Resultados}

A partir de los datos descriptivos y la comparación de los resultados relacionales, se determinó que el fenómeno central en el presente estudio fueron los 'significados asociados a las disciplinas de las carreras de la salud', lo que permite ganar lucidez respecto a lo que los docentes entienden por disciplina científica y el desarrollo de la didáctica en ciencias de la salud (Figura). Para esclarecer el marco referencial, se entenderá como didáctica aquellos aspectos que armonizan con la naturaleza disciplinar de cada carrera de la salud, es decir, su contenido teórico disciplinar y las formas específicas de entender qué es enseñar y qué es aprender [10]. Siguiendo la propuesta integral de la teoría fundamentada [13], ello puede evidenciarse en la figura.

En primer lugar, se revela como contexto educativo-político chileno una suerte de desautonomización de las instituciones de educación superior [18], debido a su condición de regulación por la Comisión Nacional de Acreditación, marco desde el cual intentan responder prioritariamente a los requerimientos de dicha comisión [19], lo que se traduce en una desconexión entre universidad y comunidad (Tabla I). En segundo lugar, los docentes están inmersos en un contexto que no propicia condiciones que aseguren un mejor desempeño en el quehacer educativo. En un análisis descriptivo realizado en este proyecto se observaron seis condiciones mínimas (campo clínico, contexto educativo, valoración del perfil de ingreso, número de estudiantes, formación docente, recursos económicos y físicos) del
Figura. Codificación selectiva.

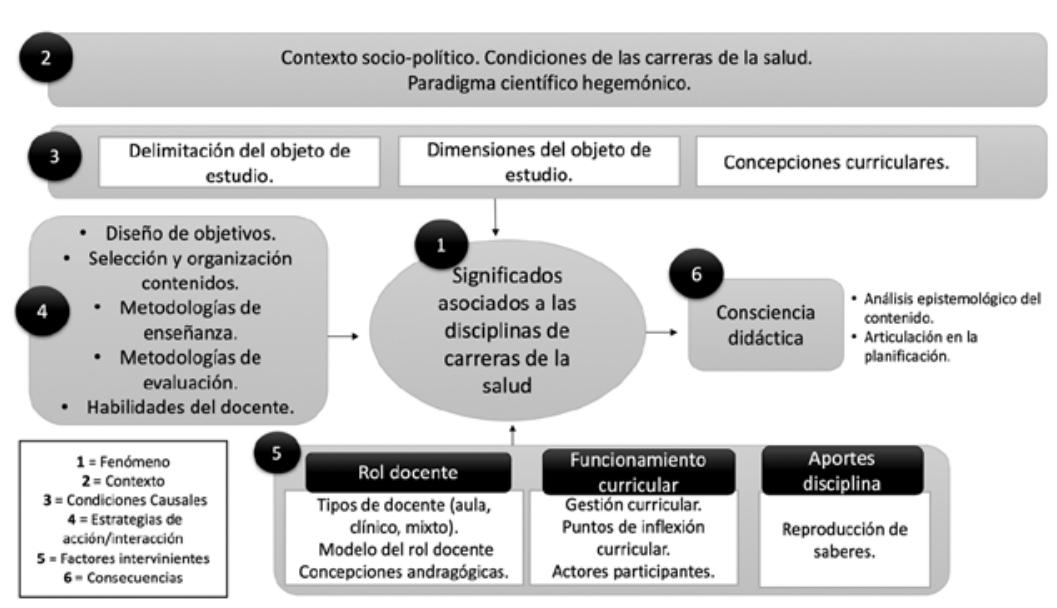

contexto educativo que deberían asegurarse para impartir una enseñanza de calidad y que, en la medida en que se resuelvan, permitirán influir de manera positiva o negativa en la didáctica de las disciplinas. Este desarrollo también va de la mano del modelo educativo actual de la universidad y las condiciones que ésta proporciona para la generación de conocimiento en carreras de salud, es decir, el apoyo que esta institución ofrece para la capacitación en investigación clínica, habilitación de laboratorios y contratación de equipos de trabajos [20]. Por último, se observa de cierta forma una legitimización del paradigma clínico, un paradigma hegemónico caracterizado por ser ontológicamente empirista y epistemológicamente basado en el método científico positivista, orientado a validar la ciencia de la salud como una ciencia normal (Tabla I).

Se observan ciertos antecedentes causales de este fenómeno, como es la falta de delimitación del objeto de estudio en ciencias de la salud y el desconocimiento de sus dimensiones biológicas y sociales. Actualmente, los docentes consideran el objeto de estudio en salud desde su dimensión biológica y existe escaso reconocimiento de su dimensión social, lo que influye en la conceptualización de los profesionales de la salud sobre su disciplina (Tabla I). Refieren que su disciplina es científica, pero este argumento tiende a relegar dichos atributos a asignaturas que corresponden al ciclo básico y no se menciona específicamente cuál es la naturaleza de la ciencia que enseñan, lo que lleva a interpretar que existe un problema en la delimitación del propio objeto de estudio (Tabla I). 
Tabla I. Citas asociadas a la construcción del análisis de codificación selectiva (I).

\section{Contexto Desconexión academia y comunidad:}

'La universidad pretende ir a entregar cosas y los del otro lado pretenden que la universidad les entregue, pero no hay una bidireccionalidad, no hay una cooperación, no hay cosa que a mí me gusta mucho, la colaboración. Algo, tenemos una visión en una visión de conjunto. No. Y por lo tanto, si tú no te fijas, una cosa está que, que en la malla curricular por separado. Pero también cuando intentamos articular este plan de estudios con la realidad tampoco es articulado' [entrevistado 12, doctor en Ciencias de la Educación, 49 años]
Hegemonía del pensamiento clínico:

'Bueno, si el objeto de estudio es el hombre. En toda su etapa del ciclo vital, ¿no es cierto? Y especialmente lo referente a la salud y cómo va evolucionando y cómo va haciendo la prevención y la promoción de su salud, que son los fuertes, digamos. Y es aún una parte de la colaboración médica la parte de la curación, pero el rol primordial es la parte de la promoción, la prevención de las enfermedades y la promoción de la salud del individuo y de todo el grupo comunitario' [entrevistado 13, enfermera, 68 años]

'Entonces, si uno piensa en la didáctica, bajaba su cabeza y se ponía al servicio de eso. Cuando hay una reflexión un poco mayor, cuando uno empieza, dicen las teorías cognitivas, hay un contexto histórico también, hay un contexto más crítico. Fíjate bien, Vigotsky es un crítico. Si uno piensa en Focault y todos estos que empiezan a mirar la realidad social y empiezan a ver la escuela y empiezan a ver los psiquiátricos como cárceles, levantan una lógica mucho más crítica. Como si en América latina, Pablo Freire empieza a ver cómo esta escuela bancaria' [entrevistado 12, doctor en Ciencias de la Educación, 49 años]
Antecedentes Significado de ciencia: ¿cómo se comprende la ciencia?

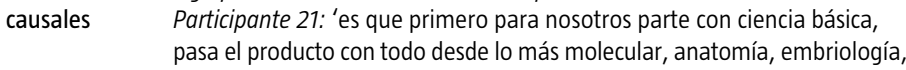
eh, después viene todo lo que es en el fondo'

Participante 20: 'morfología' [fonoaudiólogo, 44 años]

Participante 21: 'los anexos, morfología en mi caso, cómo se mueve el ojo, qué nervios, qué patologías asociadas, y luego de eso que ya tienes en teoría, vas a la práctica. La práctica obviamente es escalonada, partes idealmente viendo que es sano para saber que es enfermo y ver patologías después ya oculares, y después tratamiento y junto con los tratamientos después vienen las prácticas. sobre todo quirúrgicas' [ingeniero estadístico, 27 años]

Participante 19: 'El aprendizaje en el área del fonoaudiólogo también tuvo que ver la integración de las ciencias básicas, ya se sea la embriología; en mi tiempo también la matemática, la física y, posteriormente, la lingüística, la psicología' [enfermera, 27 años]

Participante 22: 'Yo soy como súper distinto, yo todo fue pura matemática principios, mucho cálculo, mucha algebra' [mujer, médico, 31 años] [focus group 1, docentes de carreras de la salud]
Delimitación del objeto de estudio:

'Las carreras del área de la salud las veo en un campo intermedio, además. ¿Por qué? Porque tienen mucho de las ciencias sociales, porque su objeto es un ser humano y en particular la salud, pero por otro lado tiene harto del campo de las ciencias naturales. Entonces más complicado todavía, porque hay como una convivencia, por así decirlo, que no sé hasta qué punto puede ser tan positiva entre esos dos objetos' [entrevistado 12, doctor en Ciencias de la Educación, 49 años]

'La idiosincrasia va mucho más (...) amplia que la de un adulto. El otro tiene la vida resuelta, lo que pasa es que le pasó en el camino que tuvo un problema. En el niño no, en el niño no, todavía no sabemos, no ha desarrollado su potencial neurológico, sensorial, falta mucho todavía, entonces es mucho más complejo' [entrevistado 16, fonoaudióloga, 32 años]
Un experto en didáctica señaló que, considerando la evolución de las carreras de salud, éstas comenzaron a supeditarse a un ámbito disciplinar intermedio entre las ciencias biológicas y las ciencias sociales (Tabla I). Sin embargo, los docentes no tienen certeza de esto. Ello tiene relación directa con el conocimiento que los docentes poseen respecto a su disciplina científica [4].

Como tercer antecedente contextual, se identificó que tanto el modelo como la estructura curricular se caracterizan por ser rígidos en su división de ciclo básico y clínico. Este tipo de currículo evidencia de cierta manera cómo los docentes están concibiendo lo que es enseñar y aprender, lo que se vio en resultados preliminares [3].

Ahora bien, siguiendo el análisis y respecto a las estrategias de acción e interacción, se observó que lo mencionado con anterioridad ha determinado por qué los docentes en carreras de salud despliegan una serie de acciones y habilidades para enseñar, co- mo es el diseño de objetivos, la selección y organización de los contenidos de enseñanza, y la selección y organización de metodologías de enseñanza y evaluación $[4,5]$. Sin embargo, se ha producido una suerte de mecanización rígida de la gestión didáctica, en la cual el docente escoge y configura criterios arbitrarios de lo que significa enseñar en su disciplina científica, lo que se traduce en determinadas formas de organizar los contenidos, el consenso en los criterios de enseñanza o cómo se piensa la vinculación entre teoría y práctica (Tabla II).

Paralelamente, emergen una serie de factores que intervienen en el fenómeno de estudio y que en la actualidad influyen en el desarrollo de la dimensión didáctica. Ello tiene relación con el tipo de docentes y el papel que desempeñan en su práctica educativa diaria, el modelo y concepciones andragógicas. Por otra parte, el funcionamiento curricular que se está llevando a cabo en las carreras de salud aún es desconocido, a pesar de los esfuerzos por diseñar 
Tabla II. Citas asociadas a la construcción del análisis de codificación selectiva (II).

\begin{tabular}{ll}
\hline Estrategias de & Falta de consenso en los criterios de enseñanza de los docentes: \\
acción/interacción & 'Parece que se hacen reuniones con estos docentes, por lo menos una \\
& vez al año, pero a solicitud de nosotros. Entonces, supón que nosotros \\
& decimos, ¿sabe qué?, consideramos que los alumnos no están llegando \\
& con los conocimientos' [entrevistado 4, tecnóloga médica, 58 años] \\
& Organización de contenidos: \\
& 'Eso tiene que ver con lo que iba a comentar yo, que depende mucho de \\
& quién sea el encargado de la asignatura para la respectiva carrera, incluso \\
& de año a año a veces por razones $X$ cambia y por ende la orientación o el \\
& peso que le da a los contenidos varía completamente. Y lo otro es que, en \\
& las partes, en los grupos prácticos, también está como muy individualizado, \\
no es como una cosa, aunque aparentemente en el primer ciclo no hay & como unos objetivos de aprendizaje específicos' [entrevistado 30, \\
matrona, 53 años] [focus group 2]
\end{tabular}

Cómo pensar la vinculación teórico-práctica:

'Los procesos de reflexión o los procesos de aprendizaje de ciertas cosas, si uno las compara en el aula y las compara en los centros de práctica, tienen una cosa en común y la cuestión en común es que es la articulación entre la teoría y la práctica, y no se dé en el aula. En el aula a lo mejor puedes hacer una relación entre la teoría y la práctica desde la representación. No sé si me explico, es cuando el profesor está hablando de su experiencia, trae a colación una experiencia propia o hace que los alumnos traigan a colación en su cabeza una experiencia personal. Pero, fíjate bien, en el plano de las representaciones, en cambio, cuando está en la sala de urgencias, esa articulación es real. Y ahí se juegan las cosas, se juega la verdad de lo que has aprendido, de cómo lo solucionas y cómo eso da oportunidades' [entrevistado 12, doctor en Ciencias de la Educación, 49 años]

\section{Factores}

intervinientes

\begin{abstract}
Concepción de contar con las ciencias básicas para poder nivelar los conocimientos:
'Yo encuentro que igual es necesario tener como algo básico porque ocurren algunos procesos fisiológicos, porque es importante poder, conocer la célula, cosas básicas' [entrevistado 5, tecnóloga médica, 31 años]

Funcionamiento curricular:

'Nuestra carrera es una disciplina que se sustenta en la ciencia y obviamente tiene [que tener] las bases. Muchas de las cosas que nosotros hacemos, aplicamos, de entender un poco lo que hacemos, están en las ciencias básicas. Y eso le pasa a todas las carreras. Entonces de alguna forma, como digo, el nuevo currículo que pretende (...) poner al lado de las ciencias básicas duras (...) módulos disciplinares de la disciplina. Entonces de esa manera se puede articular, se puede integrar un poquito mejor o entender un poco mejor' [entrevistado 17, cinesiólogo, 47 años]

Rol de los docentes:

'En teoría, en el papel, se intenta poner lo que la carrera quiere, pero las ciencias básicas en general en nuestra universidad hacen lo que les parece (...). Y depende del docente de turno en realidad si se profundiza o no en el ámbito que la carrera particular pide, y la metodología general yo creo que se considera una asignatura que es para todas las carreras; creo que ocupa la misma metodología, teórico y después tienen práctico' [entrevistado 31, médico, 55 años] [focus group 2]
\end{abstract}

\section{Aportes de la disciplina:}

'El aporte de la carrera fundamentalmente es mejorar el diagnóstico clínico, porque muchas cosas antes se diagnosticaban empíricamente en base a la experiencia del médico. En cambio ahora, con el avance de la tecnología, uno puede dirigir el diagnóstico' [entrevistado 4, tecnólogo médico, 58 años] Evolución profesional:

'El cinesiólogo no tenía esta proyección que tiene hoy en día, de que a pesar de que los cinesiólogos no están sólo en lo privado, trabajan, hacen ejercicio libre de su profesión y no están remitidos solamente a lo de antes, que el cinesiólogo debía trabajar en un hospital público' [entrevistado 11, cinesiólogo, 69 años] Aportes de otras disciplinas:

'De la enfermería yo diría que la psicología, la antropología, la sociología, o sea todas estas ciencias de las humanidades que llamamos ciencias sociales, yo diría que han contribuido muy fuerte porque dan un conocimiento en relación a cómo el hombre reacciona como individuo o cómo reacciona en masa en las comunidades desde el punto de vista de la sociología y la antropología; también, cómo se vive dentro de la cultura del hombre. Yo creo que es primordial y puede ser que la enfermería ha ido como un poco cambiando. Si antes se hablaba de que eran pertenecientes a las ciencias médicas, ahora se habla de que son de las ciencias sociales netamente' [entrevistado 13, enfermera, 68 años] instrumentos que permitan evaluar la gestión curricular (Tabla II). Además, las características de las disciplinas, algunas de las cuales son más jóvenes que otras, difieren en su promoción de conocimiento en Chile y, por tanto, deben destinarse esfuerzos en gestionar recursos de manera distinta. Actualmente, dichas áreas del conocimiento se ven nutridas no solo por las ciencias bioquímicas, químicas, físicas y de la ingeniería, principalmente, sino también por disciplinas de las ciencias sociales. Esto daría cuenta de que existen disciplinas que derivan conocimiento a las ciencias de la salud (Tabla II). De esa manera se observa que el desarrollo de la disciplina, es decir, la evolución en el tipo de intervenciones clínicas, instrumentos diagnósticos y rol profesional, también interfiere en la conceptualización que se vaya a hacer de las ciencias de la salud.

Considerando todos estos elementos, se pueden evidenciar ciertas consecuencias que emergen de este fenómeno. La identificación y reconocimiento de los significados particulares que un docente puede atribuir a su disciplina científica tendrá una influencia en el proceso de concienciación didáctica, lo que le permitirá articular adecuadamente o no sus estrategias de enseñanza, en la evaluación y en un diseño coherente o incoherente de organización del contenido. Lo anterior permitirá tener una guía clara respecto a cómo debe darse la progresión de los aprendizajes clínicos en su naturaleza teórica y práctica (Tabla II).

\section{Discusión}

En la universidad hemos destinado esfuerzos a abordar el fenómeno del aprendizaje y la enseñanza en carreras de salud, lo que ha sido valioso para promover mejoras educativas. Sin embargo, creemos 
que es fundamental destinar también esfuerzos en analizar de qué forma se está pensando la vinculación que existe entre ciencia y enseñanza, que son los pilares de la formación profesional en la educación superior del siglo XXI $[7,8,12]$. Jorba y Casellas [21] fueron enfáticos en este punto al mencionar que era urgente y necesario profundizar en el conocimiento del contenido de la materia que se enseña, considerando que es principalmente la reflexión epistemológica de las ciencias uno de los medios por los cuales se puede pensar un contenido disciplinar en la enseñanza. No queremos decir con esto que los docentes no deban formarse en competencias educativas, sino que además de dicho esfuerzo, deben ser parte fundamental del proceso reflexivo científico de las disciplinas que enseñan. De lo contrario, sucederá que los docentes instalarán acciones como meros reproductores de conocimiento científico [5], y si no son agentes activos en el proceso de reflexión crítica de su ciencia, difícilmente lo serán de su didáctica (Figura). Por esta razón, se estima necesario abrir nuevos campos de reflexión en educación en ciencias de la salud que vinculen el saber pedagógico con el científico y que se discuta de qué forma se orientará la enseñanza en términos ontológicos y epistémicos.

En el siglo xx comenzaron a sentarse fuertemente las tensiones sobre la idea de seguir pensando la ciencia a partir de metarrelatos universales, herencia de un pensamiento moderno, y comenzó a emerger una búsqueda por lo particular, lo ideográfico, lo contextual, social y cultural $[22,23]$. Dicha discusión ha generado esfuerzos argumentativos en diversas ciencias del conocimiento, pero parece que la educación de ciencias de la salud ha permanecido ajena a dichas problemática. La pregunta de por qué hemos estados ajenos a la discusión que se hace en el pensamiento científico contemporáneo encuentra su reverberación en la legitimación de verdades y dispositivos que se inserta en el ámbito de la salud [23,24]. Esto se relaciona con la significación que entienden los docentes respecto de su disciplina como ciencia normal y hegemónica. A partir de los resultados evidenciados, vemos que los docentes logran identificar que la naturaleza del objeto de estudio en ciencias de la salud tiene al menos dos dimensiones: la biológica y la social (Figura; Tabla I). Entonces, tendríamos que preguntarnos: ¿cuál sería la discusión de la enseñanza científica en salud considerando la naturaleza propia del objeto de estudio?

Si bien los docentes podrían estar atentos a las discusiones científico-biológicas de su disciplina, también deberían considerar las discusiones cientí- fico-sociales. Según Ramos [25], el 'giro interpretativo' y el 'giro lingüístico' que han hecho las ciencias sociales, así como la ruptura posmodernista [23,24] y estudios poscoloniales, han creado una intensa discusión sobre el uso práctico del conocimiento científico. Esto nos hace cuestionar al menos de qué forma estamos comprendiendo al ser humano en ciencias de la salud. En cierto modo, nos hemos distanciado de la ferviente tensión que dichas discusiones generan y de la pregunta que se deriva de ellas: ¿será la finalidad de la enseñanza en ciencias de la salud el perpetuar una forma de pensar científica, al alero de las discusiones contemporáneas? Posiblemente hemos tomado una actitud pasiva y hemos pensado que la salud no forma parte de dichas discusiones y, en menor medida, la discusión de la educación en salud. Sin embargo, ¿cuánto será posible seguir distanciados de dicha discusión? Resulta problemático lo anterior, puesto que pensar en una formación profesional de carreras de salud exenta de la discusión sobre el pensamiento científico contemporáneo solo restringirá el proceso reflexivo y formativo para una educación del siglo XxI. La propuesta que logramos develar con nuestros resultados es pensar la práctica reflexiva del docente no solo desde la evaluación y la enseñanza, sino llegar a comprender y formar espacios de discusión sobre la concepción científica para así dar cuenta de las concepciones didáctica que analizan los contenidos epistémicos y ontológicos de los saberes disciplinares [10,11].

\section{Bibliografía}

1. Flexner A. The Flexner report on medical education in the United States and Canada. New York: Carnegie Foundation; 1910.

2. Rodríguez-Learte AI, González-Soltero R, Rodríguez-Martín I Sánchez A, Gal B. Liderando el cambio: hacia un currículo integrado para ciencias biomédicas. Experiencia de la Universidad Europea de Madrid. FEM 2018; 21: 215-22.

3. Ortega J, Matus O, Márquez C, Parra P, Bastías N, Arellano J, et al. Concepciones docentes respecto al currículum en carreras de la salud. Revista de Educación en Ciencias de la Salud 2017; 14: 138-43.

4. Pérez C, Fasce E, Coloma K, Vaccarezza G, Ortega J. Percepción de académicos de carreras de la salud de Chile sobre el perfeccionamiento docente. Rev Med Chile 2013; 141: 787-92.

5. Ortega J, Ortiz L, Nocetti A. Prácticas reflexivas del proceso de enseñanza-aprendizaje en docentes universitarios de las ciencias de la salud. Educación Médica Superior 2015; 29: 576-90.

6. Pincus K, Stout D, Sorensen J, Stocks K, Lawson R. Force for change in higher education and implications for the accounting academy. Journal of Accounting Education 2017; 40: 1-18.

7. Muñoz M, Garay F. La investigación como forma de desarrollo profesional docente: retos y perspectivas. Estudios Pedagógicos 2015; 41: 389-99.

8. Valencia J, Macías J, Valencia A. Formative research in higher education: some reflections. Soc Behav Sci 2015; 176: 940-5.

9. Ríos-González CM. Capacitación en investigación científica en estudiantes de medicina. FEM 2017; 20: 313. 
10. Grossman P, Wilson S, Shulman L. Profesores de sustancia: el conocimiento de la materia para la enseñanza. Profesorado, Revista de Currículum y Formación de Profesorado 2005; 9: 1-25.

11. Chevallard Y. La transposición didáctica: del saber sabio al saber enseñado. Buenos Aires: Aique; 2000.

12. Hernández I. El docente investigador en la formación de profesionales. Revista Virtual Universidad Católica del Norte 2009; 27: 1-21.

13. Strauss A, Corbin J. Bases de la investigación cualitativa. Técnicas y procedimientos para desarrollar la teoría fundamentada. Medellín: Universidad de Antioquia; 2002.

14. Rodríguez G, Gil J, García E. Metodología de la investigación cualitativa. Málaga: Aljibe; 1999.

15. Vieytes R. De la investigación en organizaciones, mercado y sociedad. Buenos Aires: Editorial de las Ciencias; 2004.

16. Patton M. Qualitative evaluation methods. Beverly Hills, CA: Sage Publications; 1980

17. Guba E, Lincoln Y. Paradigmas en competencia en la investigación cualitativa. In Denman C, Haro JA, eds. Por los rincones. Antología de métodos cualitativos en la investigación social. Sonora: Colegio de Sonora; 2002. p. 113-45.

18. Rama C. La tendencia a la masificación de cobertura de la educación superior en América Latina. Revista Iberoamericana de Educación 2009; 50: 173-95.

19. Ministerio de Educación. Criterios de evaluación para la acreditación de carreras profesionales con licenciatura y programas de licenciatura. Santiago, Chile: Comisión Nacional de Acreditación (CNA); 2015. URL: https://www.cnachile.cl/ Paginas/Inicio.aspx.

20. Matus O, Ortega J, Parra P, Ortiz L, Márquez C, Stotz M, et al. Condiciones del contexto educativo para ejercer el rol docente en ciencias de la salud. Un enfoque cualitativo. Rev Med Chile 2017; 145: 790-7.

21. Jorba J, Casellas E. Estrategias y técnicas para la gestión social en el aula. Barcelona: Síntesis; 1997.

22. Foucault M. La arqueología del saber. Buenos Aires: Siglo XXI; 2011.

23. Lyotard J. La condición postmoderna. Informe sobre el saber Buenos Aires: Editorial REI; 2000.

24. Foucault M. El nacimiento de la clínica. Una arqueología de la mirada médica. Buenos Aires: Siglo XXI; 2008.

25. Ramos C. El ensamblaje de ciencia social y sociedad. Conocimiento científico, gobierno de las conductas y producción de lo social. Santiago de Chile: Ediciones Universidad Alberto Hurtado; 2012. 
